\title{
Nuclear lattice simulations
}

\author{
Dean Lee ${ }^{1, a}$, Evgeny Epelbaum²,3 ${ }^{2, H e r m a n n}$ Krebs $^{3}$, and Ulf-G. Meißner ${ }^{3,2,4}$ \\ 1 Department of Physics, North Carolina State University, Raleigh, NC 27695, USA \\ 2 Institut für Kernphysik (IKP-3) and Jülich Center for Hadron Physics, Forschungszentrum Jülich, D-52425 Jülich, Ger- \\ many \\ 3 Helmholtz-Institut für Strahlen- und Kernphysik (Theorie) and Bethe Center for Theoretical Physics, Universität Bonn, \\ D-53115 Bonn, Germany \\ 4 Institute for Advanced Simulation (IAS), Forschungszentrum Jülich, D-52425 Jülich, Germany
}

\begin{abstract}
We review recent progress on nuclear lattice simulations using chiral effective field theory. We discuss lattice results for dilute neutron matter at next-to-leading order, three-body forces at next-to-next-toleading order, isospin-breaking and Coulomb effects, and the binding energy of light nuclei.
\end{abstract}

\section{Introduction}

Nuclear lattice simulations based on low-energy effective field theory have been used to study nuclear matter [1] and neutron matter [2-7]. The method has also been applied to very light nuclei $A \leq 4$ in pionless effective field theory [8] and chiral effective field theory at leading order (LO) [9]. More recently next-to-leading order (NLO) calculations have been carried out for the ground state of neutron matter [10,11]. A review of lattice methods and results using effective field theory can be found in Ref. [12]. In this proceedings article we focus on several recent calculations since the writing of the review article.

\section{Leading order}

At leading order in chiral effective field theory the nucleonnucleon effective potential is

$$
V_{\mathrm{LO}}=V+V_{I^{2}}+V^{\mathrm{OPEP}} .
$$

$V, V_{I^{2}}$ are the two independent contact interactions at leading order in the Weinberg power counting scheme, and $V^{\mathrm{OPEP}}$ is the instantaneous one-pion exchange potential. The interactions in $V_{\mathrm{LO}}$ can be described in terms of their matrix elements with two-nucleon momentum states. In the following $\mathbf{q}$ denotes the $t$-channel momentum transfer while $\mathbf{k}$ is the $u$-channel exchanged momentum transfer. We use $\tau$ to represent Pauli matrices in isospin space and $\sigma$ for Pauli matrices in spin space.

For the two leading-order contact interactions the amplitudes are

$$
\begin{gathered}
\mathcal{A}(V)=C, \\
\mathcal{A}\left(V_{I^{2}}\right)=C_{I^{2}} \tau_{A} \cdot \tau_{B} .
\end{gathered}
$$

\footnotetext{
${ }^{a}$ e-mail: dean_lee@ncsu.edu
}

For the one-pion exchange potential,

$$
\mathcal{A}\left(V^{\mathrm{OPEP}}\right)=-\left(\frac{g_{A}}{2 f_{\pi}}\right)^{2} \frac{\left(\boldsymbol{\tau}_{A} \cdot \boldsymbol{\tau}_{B}\right)\left(\mathbf{q} \cdot \boldsymbol{\sigma}_{A}\right)\left(\mathbf{q} \cdot \boldsymbol{\sigma}_{B}\right)}{q^{2}+m_{\pi}^{2}} .
$$

Here $m$ is the nucleon mass, $m_{\pi}$ the pion mass, $f_{\pi}$ the pion decay constant, and $g_{A}$ the nucleon axial charge.

In Ref. [9] two different lattice actions were considered which were later denoted $\mathrm{LO}_{1}$ and $\mathrm{LO}_{2}$ [10]. The interactions in $V_{\mathrm{LO}_{1}}$ include one-pion exchange and two zerorange contact interactions corresponding with amplitude

$$
\mathcal{A}\left(V_{\mathrm{LO}_{1}}\right)=C+C_{I^{2}} \tau_{A} \cdot \tau_{B}+\mathcal{A}\left(V^{\mathrm{OPEP}}\right) .
$$

The interactions in $V_{\mathrm{LO}_{2}}$ consist of one-pion exchange and two Gaussian-smeared contact interactions,

$$
\mathcal{A}\left(V_{\mathrm{LO}_{2}}\right)=C f(\mathbf{q})+C_{I^{2}} f(\mathbf{q}) \tau_{A} \cdot \boldsymbol{\tau}_{B}+\mathcal{A}\left(V^{\mathrm{OPEP}}\right),
$$

where $f(\mathbf{q})$ is a lattice approximation to a Gaussian function. The smeared interactions in $\mathrm{LO}_{2}$ were used to better reproduce $S$-wave phase shifts for nucleon momenta up to the pion mass. In Ref. [10] phase shifts were calculated for these two lattice actions using the spherical wall method [13].

In calculations of few- and many-nucleon systems, this "improved" leading-order action is treated nonperturbatively while higher-order interactions are included as a perturbative expansion in powers of momentum, $Q / \Lambda$. This is sketched in Fig. 1.

\section{Next-to-leading order}

At next-to-leading order (NLO) the two-nucleon effective potential contains seven independent contact terms carrying two powers of momentum, corrections to the two LO 
EPJ Web of Conferences

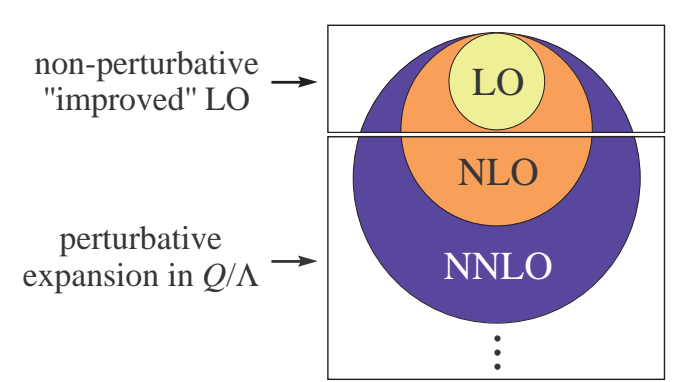

Fig. 1. The "improved" LO action is iterated non-perturbatively while the remaining higher-order interactions are treated using perturbation theory.

contact terms, and the leading contribution from the instantaneous two-pion exchange potential (TPEP) [14-18],

$$
V_{\mathrm{NLO}}=V_{\mathrm{LO}}+\Delta V^{(0)}+V^{(2)}+V_{\mathrm{NLO}}^{\mathrm{TPEP}} .
$$

The tree-level amplitudes for the contact interactions are

$$
\mathcal{A}\left(\Delta V^{(0)}\right)=\Delta C+\Delta C_{I^{2}} \tau_{A} \cdot \tau_{B}
$$

and

$$
\begin{aligned}
\mathcal{A}\left(V^{(2)}\right) & =C_{1} q^{2}+C_{2} k^{2}+\left(C_{3} q^{2}+C_{4} k^{2}\right)\left(\sigma_{A} \cdot \sigma_{B}\right) \\
& +i C_{5} \frac{1}{2}\left(\sigma_{A}+\sigma_{B}\right) \cdot(\mathbf{q} \times \mathbf{k}) \\
& +C_{6}\left(\sigma_{A} \cdot \mathbf{q}\right)\left(\sigma_{B} \cdot \mathbf{q}\right)+C_{7}\left(\sigma_{A} \cdot \mathbf{k}\right)\left(\sigma_{B} \cdot \mathbf{k}\right)
\end{aligned}
$$

The amplitude for the NLO two-pion exchange potential is $[19,20]$

$$
\begin{gathered}
\mathcal{A}\left(V_{\mathrm{NLO}}^{\mathrm{TPEP}}\right)=-\frac{\boldsymbol{\tau}_{A} \cdot \boldsymbol{\tau}_{B}}{384 \pi^{2} f_{\pi}^{4}} L(q)\left[4 m_{\pi}^{2}\left(5 g_{A}^{4}-4 g_{A}^{2}-1\right)\right. \\
\left.+q^{2}\left(23 g_{A}^{4}-10 g_{A}^{2}-1\right)+\frac{48 g_{A}^{4} m_{\pi}^{4}}{4 m_{\pi}^{2}+q^{2}}\right] \\
-\frac{3 g_{A}^{4}}{64 \pi^{2} f_{\pi}^{4}} L(q)\left[\left(\mathbf{q} \cdot \boldsymbol{\sigma}_{A}\right)\left(\mathbf{q} \cdot \boldsymbol{\sigma}_{B}\right)-q^{2}\left(\boldsymbol{\sigma}_{A} \cdot \boldsymbol{\sigma}_{B}\right)\right],
\end{gathered}
$$

where

$$
L(q)=\frac{1}{2 q} \sqrt{4 m_{\pi}^{2}+q^{2}} \ln \frac{\sqrt{4 m_{\pi}^{2}+q^{2}}+q}{\sqrt{4 m_{\pi}^{2}+q^{2}}-q} .
$$

On the lattice the ultraviolet momentum cutoff is inversely proportional to the lattice spacing, $\Lambda=\pi / a$. In fewand many-nucleon calculations where we use Euclideantime projection and auxiliary-field Monte Carlo, severe numerical problems appear when $\Lambda$ is very large. To avoid these problems we consider lattice simulations where the cutoff momentum is only a few times the pion mass. For the lattice calculations discussed here we take $\Lambda=314$ $\mathrm{MeV} \approx 2.3 m_{\pi}$, corresponding with $a^{-1}=100 \mathrm{MeV}$. For $|q|<\Lambda$ we can expand the two-pion exchange potential in powers of $q^{2} /\left(4 m_{\pi}^{2}\right)$,

$$
L(q)=1+\frac{1}{3} \frac{q^{2}}{4 m_{\pi}^{2}}+\cdots,
$$

$$
\begin{aligned}
& \frac{4 m_{\pi}^{2}}{4 m_{\pi}^{2}+q^{2}} L(q)=1-\frac{2}{3} \frac{q^{2}}{4 m_{\pi}^{2}}+\cdots, \\
V_{\mathrm{NLO}}^{\mathrm{TPEP}} & =-\frac{\boldsymbol{\tau}_{A} \cdot \boldsymbol{\tau}_{B}}{384 \pi^{2} f_{\pi}^{4}}\left[4 m_{\pi}^{2}\left(8 g_{A}^{4}-4 g_{A}^{2}-1\right)\right. \\
& \left.+\frac{2}{3} q^{2}\left(34 g_{A}^{4}-17 g_{A}^{2}-2\right)+O\left(\left(\frac{q^{2}}{4 m_{\pi}^{2}}\right)^{2}\right)\right] \\
& -\frac{3 g_{A}^{4}}{64 \pi^{2} f_{\pi}^{4}}\left[\left(\mathbf{q} \cdot \boldsymbol{\sigma}_{1}\right)\left(\mathbf{q} \cdot \boldsymbol{\sigma}_{2}\right)-q^{2}\left(\boldsymbol{\sigma}_{1} \cdot \boldsymbol{\sigma}_{2}\right)\right] \\
& \times\left[1+O\left(\frac{q^{2}}{4 m_{\pi}^{2}}\right)\right] .
\end{aligned}
$$

This expansion fails to converge only for values of $q$ near the cutoff scale $\Lambda \approx 2.3 m_{\pi}$, where the effective theory is already problematic due to large cutoff effects. Therefore the full non-local structure of $V_{\mathrm{NLO}}^{\mathrm{TPEP}}$ is not necessary. Instead we simply use

$$
\begin{gathered}
V_{\mathrm{LO}}=V^{(0)}+V^{\mathrm{OPEP}}, \\
V_{\mathrm{NLO}}=V_{\mathrm{LO}}+\Delta V^{(0)}+V^{(2)},
\end{gathered}
$$

where the terms in Eq. (14) with up to two powers of $q$ are absorbed as a redefinition of the coefficients $\Delta V^{(0)}$ and $V^{(2)}$. This same approach can be applied to the two-pion exchange potential at next-to-next-to-leading order (NNLO) and higher-order $n$-pion exchange potentials.

\section{Dilute neutrons at next-to-leading order}

In Ref. [11] the ground state energy for dilute neutron matter was computed using the lattice action $\mathrm{LO}_{2}$ and auxiliaryfield Monte Carlo. Next-to-leading-order corrections to the energy were also calculated. In this calculation the largest source of systematic error was the large size of NLO corrections for Fermi momenta larger than $100 \mathrm{MeV}$. The problem is caused by attractive $P$-wave interactions introduced by Gaussian smearing in $\mathrm{LO}_{2}$ that must be cancelled by NLO corrections. In systems with both protons and neutrons this $P$-wave correction is numerically small when compared with the strong binding produced by $S$-wave interactions. For pure neutron matter, however, the $S$-wave interactions produce much less binding due to Fermi repulsion. Therefore on a relative scale, the $P$-wave interactions are not as small an effect in neutron matter.

These problems were resolved using a new leadingorder action $\mathrm{LO}_{3}$ [21]. The interactions in $V_{\mathrm{LO}_{3}}$ correspond with the amplitude,

$$
\begin{aligned}
\mathcal{A}\left(V_{\mathrm{LO}_{3}}\right) & =C_{S=0, I=1} f(\mathbf{q})\left(\frac{1}{4}-\frac{1}{4} \sigma_{A} \cdot \sigma_{B}\right)\left(\frac{3}{4}+\frac{1}{4} \tau_{A} \cdot \tau_{B}\right) \\
& +C_{S=1, I=0} f(\mathbf{q})\left(\frac{3}{4}+\frac{1}{4} \sigma_{A} \cdot \sigma_{B}\right)\left(\frac{1}{4}-\frac{1}{4} \tau_{A} \cdot \tau_{B}\right) \\
& +\mathcal{A}\left(V^{\mathrm{OPEP}}\right) .
\end{aligned}
$$

The Gaussian-smeared interactions are multiplied by spin and isospin projection operators. Only the $C_{S=0, I=1}$ term 


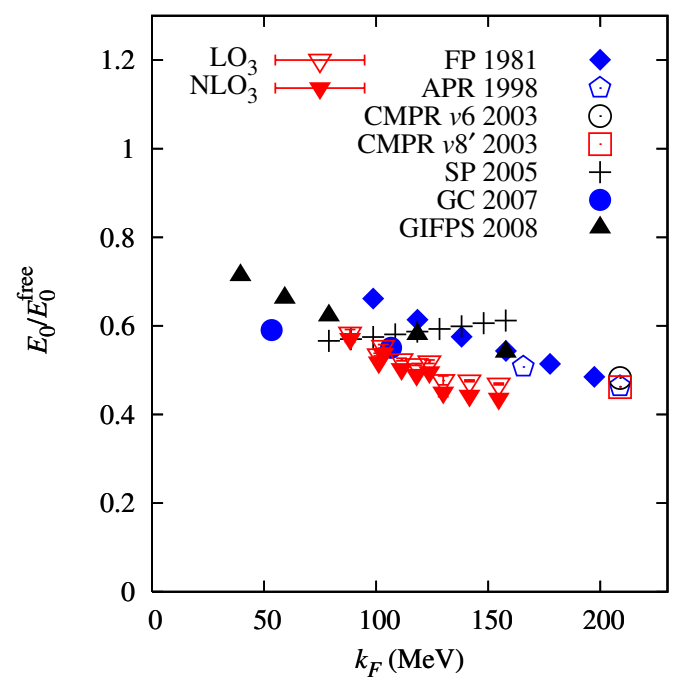

Fig. 2. Ground state energy ratio $E_{0} / E_{0}^{\text {free }}$ for $\mathrm{LO}_{3}$ and $\mathrm{NLO}_{3}$ versus Fermi momentum $k_{F}$. For comparison we show results for FP 1981 [25], APR 1998 [26], CMPR $v 6$ and $v 8^{\prime} 2003$ [27], SP 2005 [28], GC 2007 [29], and GIFPS 2008 [30].

contributes in pure neutron matter. Using the $\mathrm{LO}_{3}$ action with NLO corrections, the ground state energy for dilute neutrons has been computed in a periodic box [21].

The calculation is done using auxiliary-field Monte Carlo. One auxiliary field is associated with the total nucleon density $N^{\dagger} N$ and three fields for the spin density $N^{\dagger} \sigma N$. A review of the auxiliary-field Monte Carlo on the lattice can be found in Ref. [12]. The properties of the ground state are extracted using Euclidean-time projection. For spatial lattice spacing $a=(100 \mathrm{MeV})^{-1}$ and temporal lattice spacing $a_{t}=(70 \mathrm{MeV})^{-1}$ simulations were done with $8,12,16$ neutrons in periodic boxes with lengths $L=4,5,6,7$ in units of the spatial lattice spacing. In Fig. 2 we show results for the ratio of the interacting ground state energy to non-interacting ground state energy, $E_{0, \mathrm{NLO}} / E_{0}^{\text {free }}$, as a function of Fermi momentum $k_{F}$. For comparison we show other results from the literature: FP 1981 [25], APR 1998 [26], CMPR $v 6$ and $v 8^{\prime}$ [27], SP 2005 [28], GC 2007 [29], and GIFPS 2008 [30].

\section{Three-body forces at next-to-next-to-leading order}

A number of different phenomenological three-nucleon potentials have been investigated in the literature [31-39]. Effective field theory provides a systematic method for estimating the relative importance of three-body interaction terms. Few-nucleon forces in chiral effective field theory beyond two nucleons were first introduced in Ref. [40]. In Ref. [41] it was shown that three-nucleon interactions at NLO cancel and three-body effects first appear at next-tonext-to-leading order (NNLO). The NNLO three-nucleon effective potential includes a pure contact potential, $V_{\text {contact }}^{(3 N)}$,

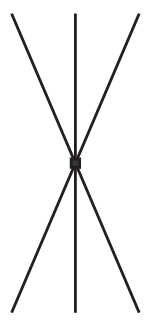

(a)

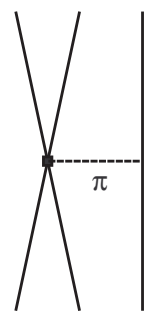

(b)

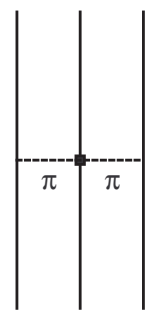

(c)
Fig. 3. Three-nucleon forces at NNLO. Diagrams (a), (b), and (c) show the contact potential, $V_{\text {contact }}^{(3 N)}$, one-pion exchange potential $V_{\mathrm{OPE}}^{(3 N)}$, and two-pion exchange potential $V_{\mathrm{TPE}}^{(3 N)}$.

one-pion exchange potential, $V_{\mathrm{OPE}}^{(3 N)}$, and a two-pion exchange potential, $V_{\mathrm{TPE}}^{(3 N)}$,

$$
V_{\mathrm{NNLO}}^{(3 N)}=V_{\mathrm{contact}}^{(3 N)}+V_{\mathrm{OPE}}^{(3 N)}+V_{\mathrm{TPE}}^{(3 N)} .
$$

The corresponding diagrams are shown in Fig. 3.

Similar to our bookkeeping notation for two-nucleon interactions, we write the tree-level amplitude for threenucleon interactions with nucleons $A, B, C$. We sum over all permutations $P(A, B, C)$ of the labels, and $\mathbf{q}_{A}, \mathbf{q}_{B}, \mathbf{q}_{C}$ are defined as the differences between final and initial momenta for the respective nucleons. The amplitudes for $V_{\text {contact }}^{(3 N)}$ and $V_{\mathrm{OPE}}^{(3 N)}$ are $[42,43]$

$$
\begin{gathered}
\mathcal{A}\left[V_{\text {contact }}^{(3 N)}\right]=\frac{1}{2} E \sum_{P(A, B, C)}\left(\boldsymbol{\tau}_{A} \cdot \boldsymbol{\tau}_{B}\right), \\
\mathcal{A}\left[V_{\mathrm{OPE}}^{(3 N)}\right]=-\frac{g_{A}}{8 f_{\pi}^{2}} D \sum_{P(A, B, C)} \frac{\mathbf{q}_{A} \cdot \boldsymbol{\sigma}_{A}}{q_{A}^{2}+m_{\pi}^{2}}\left(\mathbf{q}_{A} \cdot \boldsymbol{\sigma}_{B}\right)\left(\boldsymbol{\tau}_{A} \cdot \boldsymbol{\tau}_{B}\right) .
\end{gathered}
$$

The coefficients $E$ and $D$ are both cutoff dependent. The coefficient $E$ determines the short distance interactions between three nucleons, while $D$ determines the pion coupling to two nucleons. Following the notation introduced in Ref. [43], we define dimensionless parameters $c_{E}$ and $c_{D}$ such that

$$
E=\frac{c_{E}}{f_{\pi}^{4} \Lambda_{\chi}}, \quad D=\frac{c_{D}}{f_{\pi}^{2} \Lambda_{\chi}},
$$

and we take $\Lambda_{\chi}=700 \mathrm{MeV}$.

For convenience we separately label three parts of the two-pion exchange potential,

$$
V_{\mathrm{TPE}}^{(3 N)}=V_{\mathrm{TPE} 1}^{(3 N)}+V_{\mathrm{TPE} 2}^{(3 N)}+V_{\mathrm{TPE} 3}^{(3 N)} .
$$

The corresponding amplitudes are

$$
\begin{aligned}
\mathcal{A} & {\left[V_{\mathrm{TPE} 1}^{(3 N)}\right]=\frac{c_{3}}{f_{\pi}^{2}}\left(\frac{g_{A}}{2 f_{\pi}}\right)^{2} } \\
& \times \sum_{P(A, B, C)} \frac{\left(\mathbf{q}_{A} \cdot \boldsymbol{\sigma}_{A}\right)\left(\mathbf{q}_{B} \cdot \boldsymbol{\sigma}_{B}\right)}{\left(q_{A}^{2}+m_{\pi}^{2}\right)\left(q_{B}^{2}+m_{\pi}^{2}\right)}\left(\mathbf{q}_{A} \cdot \mathbf{q}_{B}\right)\left(\boldsymbol{\tau}_{A} \cdot \boldsymbol{\tau}_{B}\right),
\end{aligned}
$$




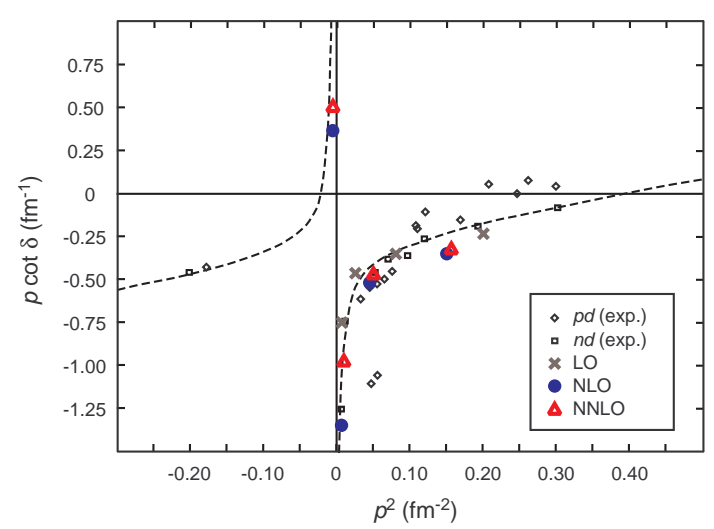

Fig. 4. Plot of $p \cot \delta$ versus $p^{2}$ for the spin-doublet channel in the center-of-mass frame. For the NNLO calculation we take $c_{D}=1.0$ fitted to the physical value for $E_{\text {triton }}$. The experimental results are from Ref. [50].

$$
\begin{aligned}
& \mathcal{A}\left[V_{\mathrm{TPE} 2}^{(3 N)}\right]=-\frac{2 c_{1} m_{\pi}^{2}}{f_{\pi}^{2}}\left(\frac{g_{A}}{2 f_{\pi}}\right)^{2} \\
& \times \sum_{P(A, B, C)} \frac{\left(\mathbf{q}_{A} \cdot \boldsymbol{\sigma}_{A}\right)\left(\mathbf{q}_{B} \cdot \boldsymbol{\sigma}_{B}\right)}{\left(q_{A}^{2}+m_{\pi}^{2}\right)\left(q_{B}^{2}+m_{\pi}^{2}\right)}\left(\boldsymbol{\tau}_{A} \cdot \boldsymbol{\tau}_{B}\right), \\
& \mathcal{A}\left[V_{\mathrm{TPE} 3}^{(3 N)}\right]=\frac{c_{4}}{2 f_{\pi}^{2}}\left(\frac{g_{A}}{2 f_{\pi}}\right)^{2} \\
& \times \sum_{P(A, B, C)}\left\{\frac{\left(\mathbf{q}_{A} \cdot \boldsymbol{\sigma}_{A}\right)\left(\mathbf{q}_{B} \cdot \boldsymbol{\sigma}_{B}\right)}{\left(q_{A}^{2}+m_{\pi}^{2}\right)\left(q_{B}^{2}+m_{\pi}^{2}\right)}\right. \\
&\left.\cdot\left[\left(\mathbf{q}_{A} \times \mathbf{q}_{B}\right) \times \boldsymbol{\sigma}_{C}\right]\left[\left(\boldsymbol{\tau}_{A} \times \boldsymbol{\tau}_{B}\right) \cdot \boldsymbol{\tau}_{C}\right]\right\} .
\end{aligned}
$$

The low-energy constants $c_{1}, c_{3}, c_{4}$ parameterize the coupling of the nucleon to two pions. These constants have been determined from fits to low-energy pion-nucleon scattering data [44], and the values $c_{1}=-0.81 \mathrm{GeV}^{-1}$, $c_{3}=-4.7 \mathrm{GeV}^{-1}, c_{4}=3.4 \mathrm{GeV}^{-1}[45]$ are used.

At fixed lattice spacing we constrain the two unknown coefficients by fitting to the triton binding energy and spin-doublet nucleon-deuteron scattering phase shifts via Lüscher's finite volume formula [46-48]. The threenucleon system is sufficiently small that we can use iterative sparse-matrix eigenvector methods to compute energy levels on cubic periodic lattices. We fix the coefficient $c_{E}$ as a function of $c_{D}$ by matching the physical triton energy at infinite volume, $-8.48 \mathrm{MeV}$. The value of the $c_{D}$ is then determined from the spin-doublet nucleon-deuteron scattering phase shifts. Results for the doublet nucleondeuteron scattering phase shift are shown in Fig. 4 using the $\mathrm{LO}_{2}$ lattice action for lattice spacing $a=(100 \mathrm{MeV})^{-1}$ and temporal lattice spacing $a_{t}=(150 \mathrm{MeV})^{-1}$ [49] with $c_{D}=1.0$.

It turns out however that the spin-doublet nucleondeuteron scattering phase shift provides only a mild constraint on $c_{D}$. Currently we are investigating other methods for constraining $c_{D}$, including one recent suggestion to determine $c_{D}$ from the triton beta decay rate [51].

\section{Isospin breaking and Coulomb effects}

In addition to isospin-symmetric interactions, we also include isospin-breaking interactions and Coulomb effects. Isospin breaking (IB) in effective field theory has been addressed in the literature [52-56]. In the following we report results from work that is currently being written for publication [57].

In the counting scheme proposed in Ref. [56], the isospin-breaking one-pion exchange interaction and Coulomb potential are numerically the same size as $O\left(Q^{2} / \Lambda^{2}\right)$ corrections at NLO. For the isospin-symmetric one-pion exchange interaction we use $m_{\pi}=m_{\pi^{0}}$ in Eq.(4). The isospin-violating one-pion exchange interaction due to pion mass differences is then

$$
\begin{gathered}
\mathcal{A}\left[V^{\mathrm{OPEP}, \mathrm{IB}}\right]=-\left(\frac{g_{A}}{2 f_{\pi}}\right)^{2}\left[\left(\tau_{1}\right)_{A}\left(\tau_{1}\right)_{B}+\left(\tau_{2}\right)_{A}\left(\tau_{2}\right)_{B}\right] \\
\times\left(\sigma_{A} \cdot \mathbf{q}\right)\left(\boldsymbol{\sigma}_{B} \cdot \mathbf{q}\right)\left[\frac{1}{q^{2}+m_{\pi^{ \pm}}^{2}}-\frac{1}{q^{2}+m_{\pi^{0}}^{2}}\right] .
\end{gathered}
$$

On the lattice we treat the Coulomb potential in position space with the usual $\alpha_{\mathrm{EM}} / r$ dependence. However this definition is singular for two protons on the same lattice site and requires short-distance renormalization via a proton-proton contact interaction. We include all possible contact interactions, namely interactions for neutronneutron, proton-proton, spin-singlet neutron-proton, and spin-triplet neutron-proton. The two neutron-proton contact interactions are already included at NLO and determined from neutron-proton scattering. The other two coefficients are determined from fitting to $S$-wave phase shifts for proton-proton scattering and the neutron-neutron scattering length. The results using the ${ }^{1} S_{0}$ neutron-proton and proton-proton phase shifts are shown in Fig. 5. The lattice data is for the $\mathrm{LO}_{3}$ action with spatial lattice spacing $a=(100 \mathrm{MeV})^{-1}$ and temporal lattice spacing $a_{t}=(150$ $\mathrm{MeV})^{-1}$. For comparison we show partial wave results from Ref. [58]. We see that the agreement is quite good for center of mass momenta up to $150 \mathrm{MeV}$.

The next results we present are for the energy splitting between helium-3 and triton. As noted already, the threenucleon system is sufficiently small that we can use iterative sparse-matrix eigenvector methods for helium-3 and the triton. We consider cubes with side lengths $L$ up to 16 fm and extract the infinite volume limit using the asymptotic result [46],

$$
E(L) \approx E(\infty)-\frac{C}{L} e^{-L / L_{0}} .
$$

Although the triton energy at infinite volume is used to set the unknown coefficient $c_{E}$, the energy splitting between helium- 3 and the triton is a prediction that can be checked. The energy difference between helium- 3 and the triton is plotted in Fig. 6 as a function of cube length. There is no significant dependence of these results upon the value of $c_{D}$. Our calculations at NNLO give a value of $0.78 \mathrm{MeV}$ in the infinite volume limit, and this agrees well with the experimental value of $0.76 \mathrm{MeV}$. 

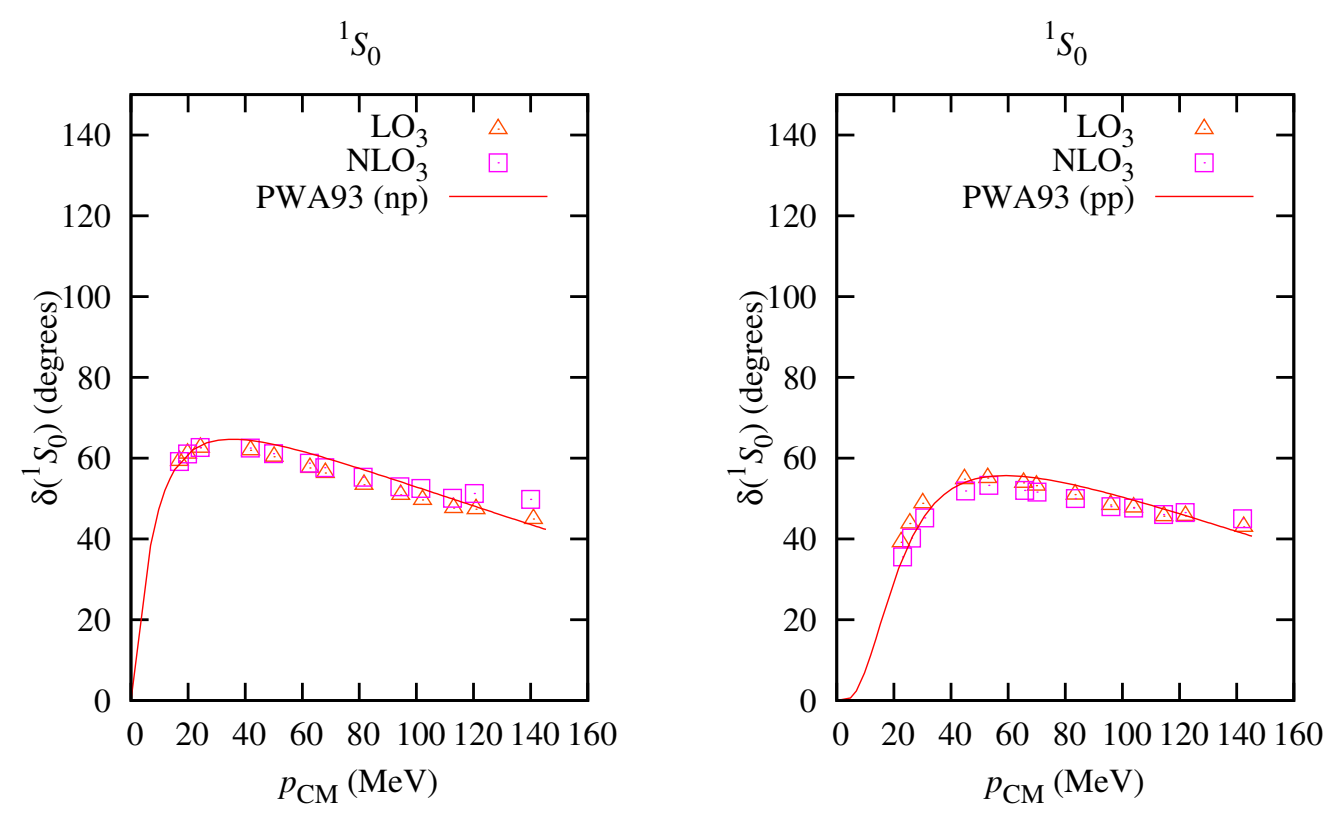

Fig. 5. ${ }^{1} S_{0}$ neutron-proton and proton-proton phase shifts versus center of mass momentum.

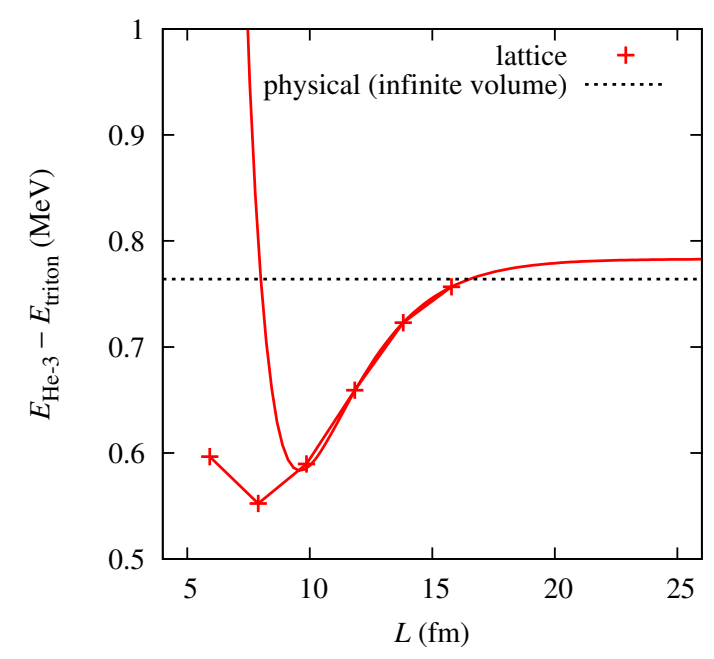

Fig. 6. The energy difference between helium- 3 and the triton versus periodic cube length.

\section{Results for nuclei $A=4,6,12$}

As in the neutron matter simulations, we use projection Monte Carlo with auxiliary fields to compute the energy of nuclei for $A \geq 4$. For the $\mathrm{LO}_{3}$ action a total of sixteen auxiliary fields are required. One auxiliary field is associated with the total nucleon density $N^{\dagger} N$, three fields for the spin density $N^{\dagger} \sigma N$, three fields for the isospin density $N^{\dagger} \tau N$, and nine fields for the spin-isospin density $N^{\dagger} \sigma \tau N$.
In the following we report results from work that is currently being written for publication [57].

In Fig. 7 we show lattice results for the ground state of helium- 4 for periodic box of length $9.9 \mathrm{fm}$. For the numerical extrapolation in Euclidean time we use the decaying exponential functions in Ref. [49]. The plot on the left shows the leading-order and higher-order contributions added cumulatively. The plot on the right shows the higher-order corrections separately. The plots show results for $c_{D}=1$. The helium-4 energy decreases about 0.4 $\mathrm{MeV}$ for each unit increase in $c_{D}$. These results are similar to those found in Ref. [49] using the $\mathrm{LO}_{2}$ action. As in that analysis we find an overbinding of about 1 to $2 \mathrm{MeV}$ at NNLO, depending on the value of $c_{D}$.

Given our cutoff momentum $\Lambda=\pi / a=314 \mathrm{MeV}$, an error of 1 to $2 \mathrm{MeV}$ is consistent with the expected size of higher order contributions. Higher order terms beyond NNLO are beyond the scope of this analysis. However if it happens that the higher-order effects are most important when all four nucleons are close to each other, then we should see universal behavior that can be reproduced by an effective four-nucleon contact interaction. We test this universality hypothesis by using an effective four-nucleon contact interaction tuned to give the physical helium-4 energy of $-28.3 \mathrm{MeV}$. The contribution of this interaction in helium-4 is shown in Fig. 7.

In Fig. 8 we show lattice results for the ground state of lithium- 6 in a periodic box of length $9.9 \mathrm{fm}$. Adding the contribution of the effective four-nucleon contact interaction to the NNLO result, we obtain an energy of -32.9 

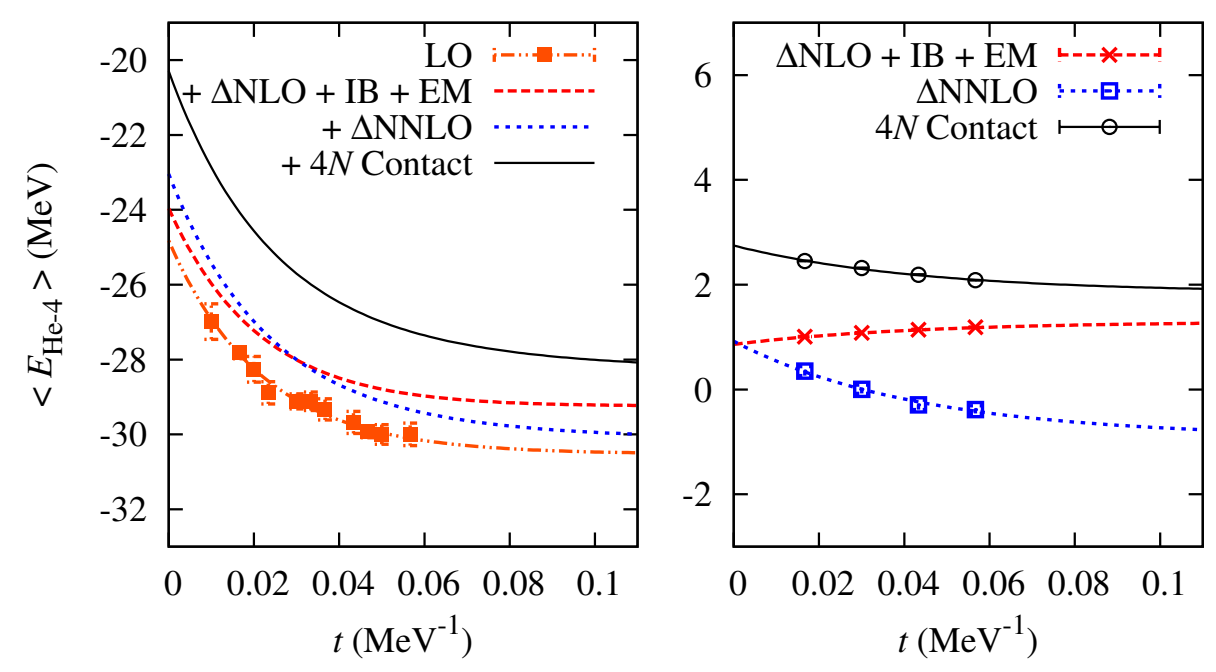

Fig. 7. Ground state energy for helium-4 as a function of Euclidean time projection.

$\mathrm{MeV}$ with an extrapolation error of about $1 \mathrm{MeV}$. This agrees quite well with the physical value of $-32.0 \mathrm{MeV}$. Extrapolation to infinite volume should move the lattice result upward slightly and closer to the physical value. The data in the plots show results for $c_{D}=1$. For each unit increase in $c_{D}$, the energy decreases about $0.7 \mathrm{MeV}$ without the four-nucleon contact interaction and about $0.3 \mathrm{MeV}$ with the effective $4 N$ contact interaction.

We show lattice results for the ground state of carbon12 in a periodic box of length $13.8 \mathrm{fm}$ in Fig. 9. Again the data in the plots show results for $c_{D}=1$. Adding the effective four-nucleon contact contribution to the NNLO result, we obtain an energy of $-99 \mathrm{MeV}$ with an extrapolation error of about $3 \mathrm{MeV}$. This is in good agreement with the physical value of $-92.2 \mathrm{MeV}$. Extrapolation to infinite volume should move the calculated value upward and closer to the physical value. For each unit increase in $c_{D}$, the energy decreases about $1.7 \mathrm{MeV}$ without the fournucleon contact interaction and about $0.3 \mathrm{MeV}$ with the effective $4 N$ contact interaction.

The results for lithium- 6 and carbon-12 support the universality hypothesis regarding higher-order interactions. More significantly the accuracy of these lattice calculations are competitive with the most accurate calculations obtain using other ab initio methods. Future lattice studies should look at decreasing the lattice spacing as well as probing large volumes. The computational scaling with the number of nucleons suggests that systems for larger nuclei are also possible.

\section{Summary}

We have presented several recent results for nuclear lattice simulations using chiral effective field theory. The method appears numerically quite promising for few-body calculations of nuclei and many-body calculations of neutron matter. At the same time, the method maintains a clear theoretical connection to effective field theory. All systematic errors are introduced up front when defining the truncated low-energy effective theory. This eliminates approximation errors tied with a specific calculational tool, physical system, or observable. By including higher-order interactions in the low-energy effective theory, one can reasonably expect systematic and systemic improvement for all lowenergy observables.

\section{Acknowledgements}

Partial financial support from the Deutsche Forschungsgemeinschaft (SFB/TR 16), Helmholtz Association (contract number VH-NG-222 and VH-VI-231), and U.S. Department of Energy (DE-FG02-03ER41260) are acknowledged. This work was further supported by the EU HadronPhysics2 project "Study of strongly interacting matter". The computational resources for this project were provided by the Jülich Supercomputing Centre at the Forschungszentrum Jülich.

\section{References}

1. H. M. Müller, S. E. Koonin, R. Seki, and U. van Kolck, Phys. Rev. C61, (2000) 044320.

2. D. Lee and T. Schäfer, Phys. Rev. C72, (2005) 024006.

3. D. Lee, B. Borasoy, and T. Schäfer, Phys. Rev. C70, (2004) 014007.

4. D. Lee and T. Schäfer, Phys. Rev. C73, (2006) 015201.

5. D. Lee and T. Schäfer, Phys. Rev. C73, (2006) 015202. 

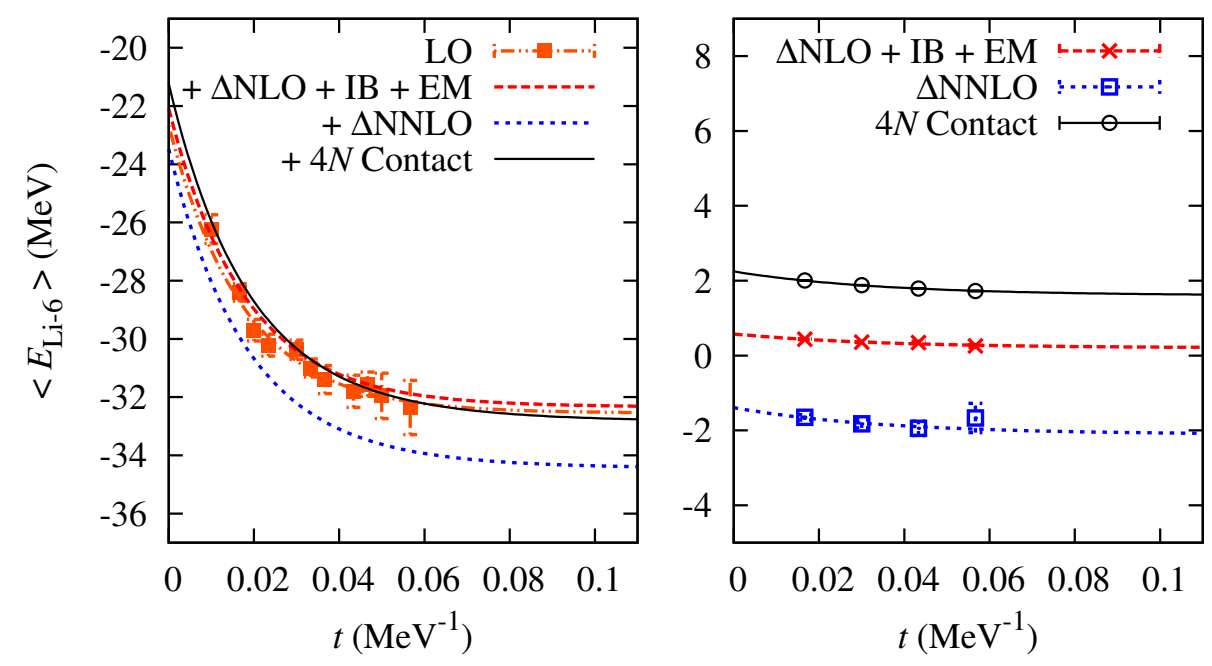

Fig. 8. Ground state energy for lithium- 6 as a function of Euclidean time projection.
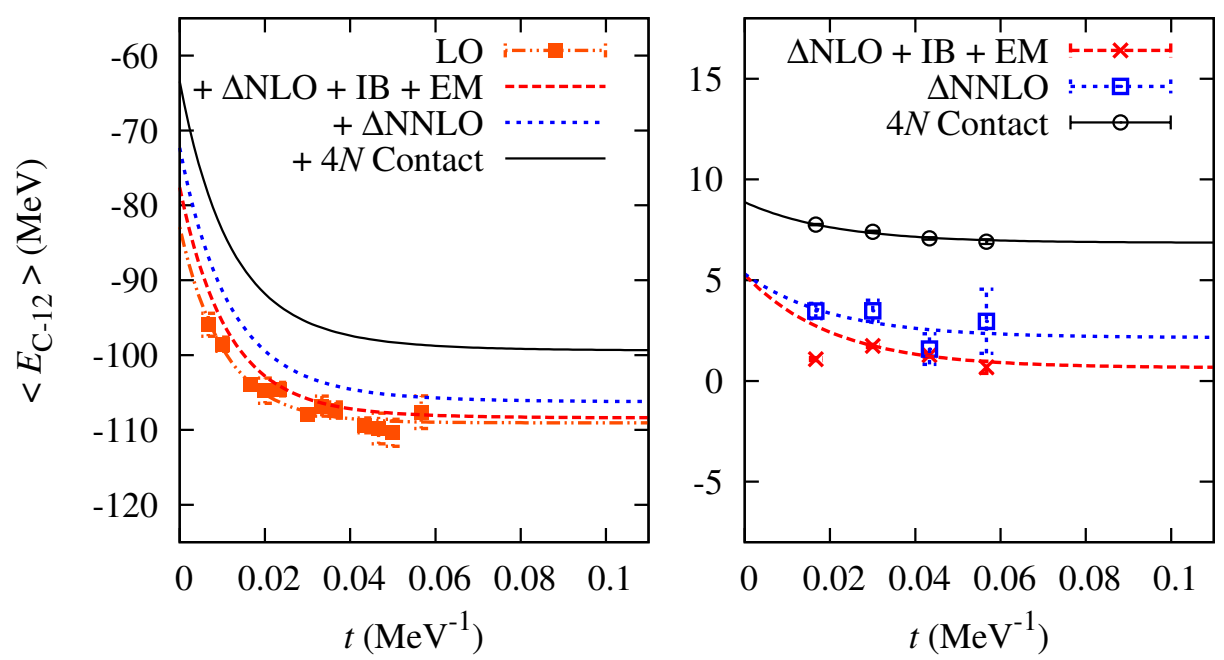

Fig. 9. Ground state energy for carbon-12 as a function of Euclidean time projection.

6. T. Abe and R. Seki, Phys. Rev. C79, (2009) 054002.

7. T. Abe and R. Seki, Phys. Rev. C79, (2009) 054003.

8. B. Borasoy, H. Krebs, D. Lee, and U.-G. Meißner, Nucl. Phys. A768, (2006) 179.

9. B. Borasoy, E. Epelbaum, H. Krebs, D. Lee, and U.-G. Meißner, Eur. Phys. J. A31, (2007) 105.

10. B. Borasoy, E. Epelbaum, H. Krebs, D. Lee, and U.-G. Meißner, Eur. Phys. J. A35, (2008) 343.

11. B. Borasoy, E. Epelbaum, H. Krebs, D. Lee, and U.-G. Meißner, Eur. Phys. J. A35, (2008) 357.

12. D. Lee, Prog. Part. Nucl. Phys. 63, (2009) 117.
13. B. Borasoy, E. Epelbaum, H. Krebs, D. Lee, and U.-G. Meißner, Eur. Phys. J. A34, (2007) 185.

14. C. Ordonez and U. van Kolck, Phys. Lett. B291, (1992) 459.

15. C. Ordonez, L. Ray, and U. van Kolck, Phys. Rev. Lett. 72, (1994) 1982.

16. C. Ordonez, L. Ray, and U. van Kolck, Phys. Rev. C53, (1996) 2086.

17. E. Epelbaum, W. Glockle, and U.-G. Meißner, Nucl. Phys. A637, (1998) 107. 
18. E. Epelbaum, W. Gloeckle, and U.-G. Meißner, Nucl. Phys. A671, (2000) 295.

19. J. L. Friar and S. A. Coon, Phys. Rev. C49, (1994) 1272.

20. N. Kaiser, R. Brockmann, and W. Weise, Nucl. Phys. A625, (1997) 758.

21. E. Epelbaum, H. Krebs, D. Lee, and U.-G. Meißner, Eur. Phys. J. A40, (2009) 199.

22. D. Lee, Phys. Rev. C71, (2005) 044001.

23. J.-W. Chen, D. Lee, and T. Schäfer, Phys. Rev. Lett. 93, (2004) 242302.

24. D. Lee, Phys. Rev. Lett. 98, (2007) 182501.

25. B. Friedman and V. R. Pandharipande, Nucl. Phys. A361, (1981) 502.

26. A. Akmal, V. R. Pandharipande, and D. G. Ravenhall, Phys. Rev. C58, (1998) 1804.

27. J. Carlson, J. Morales, J., V. R. Pandharipande, and D. G. Ravenhall, Phys. Rev. C68, (2003) 025802.

28. A. Schwenk and C. J. Pethick, Phys. Rev. Lett. 95, (2005), 160401.

29. A. Gezerlis and J. Carlson, Phys. Rev. C77, (2008) 032801.

30. S. Gandolfi, A. Y. Illarionov, S. Fantoni, F. Pederiva, and K. E. Schmidt, Phys. Rev. Lett. 101, (2008) 132501.

31. J. Fujita and H. Miyazawa, Prog. Theor. Phys. 17, (1957) 360.

32. B. H. J. McKellar and R. Rajaraman, Phys. Rev. Lett. 21, (1968) 450.

33. S.-N. Yang, Phys. Rev. C10, (1974) 2067.

34. S. A. Coon, M. D. Scadron, and B. R. Barrett, Nucl. Phys. A242, (1975) 467.

35. S. A. Coon et al., Nucl. Phys. A317, (1979) 242.

36. S. A. Coon and W. Gloeckle, Phys. Rev. C23, (1981) 1790.

37. J. Carlson, V. R. Pandharipande, and R. B. Wiringa, Nucl. Phys. A401, (1983) 59.

38. H. T. Coelho, T. K. Das, and M. R. Robilotta, Phys. Rev. C28, (1983) 1812.

39. B. S. Pudliner, V. R. Pandharipande, J. Carlson, S. C. Pieper, and R. B. Wiringa, Phys. Rev. C56, (1997) 1720.

40. S. Weinberg, Nucl. Phys. B363, (1991) 3.

41. U. van Kolck, Phys. Rev. C49, (1994) 2932.

42. J. L. Friar, D. Huber, and U. van Kolck, Phys. Rev. C59, (1999) 53.

43. E. Epelbaum, A. Nogga, W. Glöckle, H. Kamada, U.G. Meißner, and H. Witala, Phys. Rev. C66, (2002) 064001.

44. V. Bernard, N. Kaiser, and U.-G. Meißner, Int. J. Mod. Phys. E4, (1995) 193.

45. P. Büttiker and U.-G. Meißner, Nucl. Phys. A668, (2000) 97.

46. M. Lüscher, Commun. Math. Phys. 104, (1986) 177.

47. M. Lüscher, Commun. Math. Phys. 105, (1986) 153.

48. M. Lüscher, Nucl. Phys. B354, (1991) 531.

49. E. Epelbaum, H. Krebs, D. Lee, and U. G. Meißner, Eur. Phys. J. A41, (2009) 125.

50. W. T. H. van Oers and J. D. Seagrave, Phys. Lett. B24, (1967) 562.
51. D. Gazit, S. Quaglioni, and P. Navratil, Phys. Rev. Lett. 103, (2009) 102502.

52. U. van Kolck, J. L. Friar, and J. T. Goldman, Phys. Lett. B371, (1996) 169.

53. U. van Kolck, M. C. M. Rentmeester, J. L. Friar, J. T. Goldman, and J. J. de Swart, Phys. Rev. Lett. 80, (1998) 4386.

54. E. Epelbaum and U.-G. Meißner, Phys. Lett. B461, (1999) 287.

55. J. L. Friar, U. van Kolck, G. L. Payne, and S. A. Coon, Phys. Rev. C68, (2003) 024003.

56. E. Epelbaum and U.-G. Meißner, Phys. Rev. C72, (2005) 044001.

57. E. Epelbaum, H. Krebs, D. Lee, and U.-G. Meißner.

58. V. G. J. Stoks, R. A. M. Kompl, M. C. M. Rentmeester, and J. J. de Swart, Phys. Rev. C48, (1993) 792. 